\title{
Complete Digital Iconic and Textual Annotation for Mammography
}

\author{
Thomas Wittenberg $^{1}$, Matthias Elter ${ }^{1}$ and Rüdiger Schulz-Wendtland ${ }^{2}$ \\ ${ }^{1}$ Fraunhofer Institute for Integrated Circuits IIS, Erlangen \\ ${ }^{2}$ Dept. of Gynecological Radiology, University Hospital Erlangen \\ E-mail: thomas.wittenberg@iis.fraunhofer.de
}

\begin{abstract}
This work aims to propose an interactive method for a iconic and textual annotation of digital mammograms. The suggested annotation tool consists of a semantic network to represent all information about a set of mammograms obtained from experts in a structured manner based on the BIRADS standard, a correlated XML file system for persistence, data exchange and storage, and a graphical user interface, allowing a combination of iconic and textual annotation. This approach allows a complete annotation of all findings in a set of mammograms in a structured way, which is also machine readable and interpretable. Thus, systematically annotated image data sets can be used for structured information retrieval as well as case based reasoning systems for computer assisted diagnosis (CAD). Furthermore, such a digital annotation system can be used to replace paper reports and hence avoid unnecessary media breaks during the process of the examination and documentation.
\end{abstract}

\section{Introduction}

In the past years, direct digital mammography (DDM) systems have entered clinical routine and have replaced conventional screen film systems (SFS) for the acquisition of mammograms [1] in the context of screening and diagnostic processes. Nevertheless, currently in clinics worldwide, the steps to analyze the acquired digital mammograms with the goal to detect lesions such as calcifications or spiculated masses, remain the same as before. Thus, the chain of steps used to analyze the mammograms properly is related to a series of breaks between different types of media. Such breaks occur, when the digital mammogram is released on analogue film, the analogue film is viewed on a light-screen, an optical magnifying glass is used to analyze details in the detected lesions, all detected lesions are marked, noted and documented on a structured paper report, and finally the information from the paper reports are transferred back to a digital database. Along this chain of media breaks from digital images to paper reports and back to digital case databases, loss of information has to be considered. Such loss is related to information depicted in the mammograms and not recorded on the paper reports, or to information marked on the reports and not transferred completely to the digital database. Especially iconic markers on the paper formular denoting the relative positions of lesions with respect to the viewing angle cannot be transferred into the standard case databases at all. 


\section{State of the art and new contribution}

Currently, some PACS systems allow the annotation of overlay masks and markers directly into the radiographic images, which can be stored into the DICOM image files in the context of building structured reports. The drawback of this solution is, that only the iconic part of the mammogram is stored in the DICOM file, while most parts of the BIRADS documentation sheet are left undocumented. An approach collecting all information screening has been introduced by [2]. While we focus on the annotation of mammograms only, this work takes a much broader view and tries to organize the complete screening process, including ultrasound images.

One new notion of our work is the avoidance of the named media breaks during the diagnostic process between the image acquisition and the documentation. Secondly, using one single and common medium for image acquisition, annotation and storage, a consistent and complete description and documentation of a mammography examination can be obtained. Finally, such a unified and standardized documentation and annotation of mammography examinations and cases can be used to build consistent reference image data-sets.

\section{Methods}

To overcome these restrictions and media breaks, we propose the use of a digital annotation tool, which simultaneously allows the iconic annotation of regions in the images as well as a structured textual report. Such an annotation has the purpose to describe the contents of the images with respect to certain applications in a machine readable manner. For a proper documentation of mammograms, this means that all findings of lesions have to be marked and described properly. Our annotation tool consists of major components: For the representation of knowledge and facts a semantic network model is applied, implemented as a hierarchical, multi-dimensional data structure, cf. Sec. 3.1. Within a certain problem domain, this data structure organizes all related image views, the objects depicted in the images and delineated or marked by the user, as well as their individual parts and features. Secondly, a graphical user interface allows interactive annotation of image data and acquisition of application related knowledge, cf. Sec. 3.2. Finally, a XML-data structure is used for persistence, archiving and communication.

\subsection{Knowledge representation}

A possible model to organize application knowledge needed to analyse the content of images applying image processing methods as well as to represent and structure information acquired and obtained from image analysis procedures are semantic networks [3]. A semantic network can be defined as a labeled, directed and acyclic graph $\mathcal{G}=(\mathcal{V}, \mathcal{E})$. The set $\mathcal{V}=\left\{v_{1}, v_{2}, \ldots, v_{n}\right\}$ is a set of vertices (nodes) representing concepts, ideas, physical or conceptual objects, or features 
of objects. $\mathcal{E}=\left\{e_{1}, e_{2}, \ldots, e_{m}\right\}$ is a subset of $\mathcal{V} \times \mathcal{V}$ and denotes edges (or arcs) connecting ordered pairs of vertecis $\left(v_{i}, v_{j}\right) \in \mathcal{V}$. Edges denote relations between objects. The most important types of relations used within semantical networks are relations between classes and subclasses of objects such as is a, has a or has feature, and instance-relations between object-instances and object-classes. Within the scope of our activities - the image-based computer-assisted detection $(\mathrm{CAD})$ of conspicuous regions and lesions and their pre-classification - a semantic network $\mathcal{N}=(\mathcal{I}, \mathcal{V}, \mathcal{E}, \mathcal{O})$ is applied to represent knowledge depicted in and obtained from a set of images $\mathcal{I}=\left\{I_{1}, I_{2}, \ldots, I_{n}\right\}$. Since the images are usually related to a distinct imaging modality (mammography), a certain organ system (breast) and a special problem domain (mamma cancer diagnosis), a standardized ontology $\mathcal{O}$ is used to describe the corresponding image contents. For the classification of lesions depicted in mammograms, the BI-RADS (Breast Imaging - Reporting And Data System) standard of the ACR [4] is applied. Two different types of views onto a semantic network can be considered. With respect to the annotation of mammograms, where an expert describes the image contents, a knowledge-based view on the semantic network has to be taken. In this case, the vertices $v_{i}$ in the network correlate to physical objects or entities, such as the breasts, landmarks on the breast (e.g. mammilla), as well as any lesions, speculae, calcifications, clusters of micro-calcifications, etc. depicted in the images. Regarding the images from a machine vision - data-driven - point of view, the vertices represent single pixels or features, groups of pixels, sub-regions or regions, higher-level and semantic meaningful objects, as well as images or hyper-images, depicting the objects from different views. Even though different views can be taken, on a practical level the vertices of both views can be projected onto each other.

The edges $e_{i}$ denote logical or physical relations between different objects as well as relations to features of an object. In the context of mammogram annotation, the edges represent relations between different objects such as 'microcalcification $M_{i}$ belongs to calcification cluster $C_{i}$ ', 'breast $B_{\text {left }}$ has mamilla $M_{\text {left }}$ ', 'lesion $L_{i}$ is of type $B$ ', or 'lesion $L_{i}$ has textural features $c_{j}$ '.

Fig. 1 depicts two different views of the semantic network used to represent knowledge acquired from mammograms by the annotation process. The left side represents the data-driven approach, starting at the bottom with pixels and features, which are combined to form regions and objects and finally construct complete images. On the right side, the corresponding knowledge-driven approach is shown, starting with views ( $c c$ and $m l o)$ of a breast, describing its quadrants and landmarks, and finally any depicted lesions and their features.

\subsection{User interfaces}

To annotate image objects with a certain form and class, they have to be marked on the screen to provide the iconic annotation. By drawing the contours of the objects, and flood-filling them with a pre-defined class colour, each image object is annotated. In the internal data structure, the object is represented either by a binary pixel mask, or as a chain code. Both can be saved in standard image file 
Fig. 1. Overview of the organisation of the hierarchical semantic network used to describe the image contents in digital mammograms

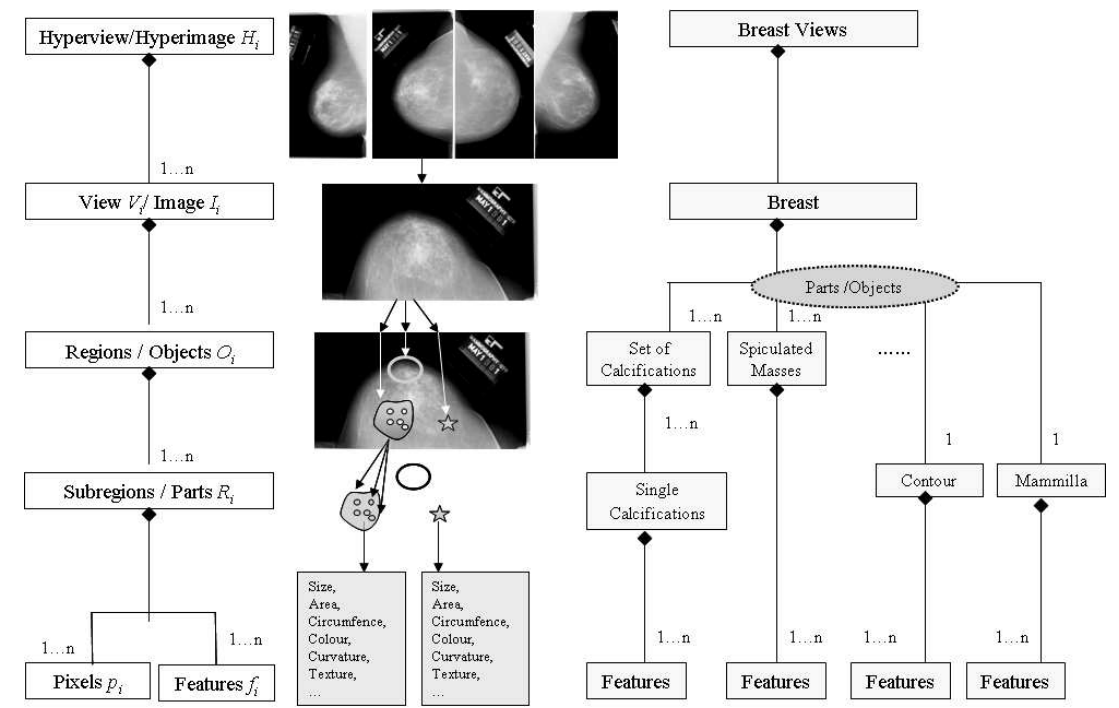

formats. If an object is depicted as several parts (e.g. a cluster of calcifications), all parts can individually be marked and then be grouped to a higher level object. If more than one image is in the data set, one physical object may have several image entities, meaning the same object can be seen from different views. E.g., usually the mammilla or a lesion are depicted from different observation angles ( $c c$ and mlo). Such connections between different entities can also be tagged and visualized. Furthermore, for each annotated lesion as well as for the complete set of images (usually 2 views of each breast), the most important parts of the BIRADS standard can be annotated using pre-defined check-boxes and radio-buttons. This part of the digital annotation mimics completely the mammography documentation sheet.

Fig. 2 shows a view of the user interface for the annotation of mammograms. The left window pane (usually depicted on an upright monitor) shows the images and the iconic annotation. The right window pane (on a second monitor) depicts the textual part of the BIRADS documentation sheet as well as the demographic data of the patient.

\section{Results}

The proposed annotation tool for digital mammograms is currently applied in the Department for Gynaecological Radiology of the University Hospital Erlangen with several goals: First, the annotation tool is used to obtain a complete and machine-readable description of the image contents of mammograms with respect to any diagnostic findings, in the context of building a reference image 
Fig. 2. User interface for the iconic and textual annotations of mammograms
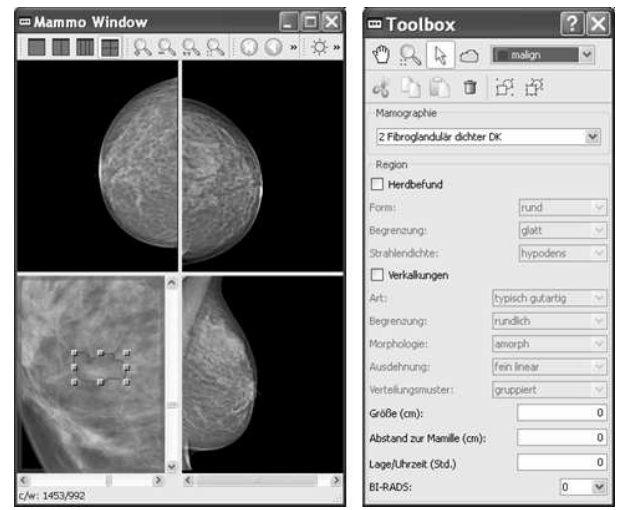

database (RID). Secondly, the thus obtained formal annotated RID will be used as a reference (gold-standard, ground truth) for the evaluation of image analysis algorithms for the automatic detection of lesions in the context of computer assisted diagnosis of mammograms. Furthermore, the RID will used for automatic information retrieval in the context of a case-based reasoning system. Currently, about 250 complete image data sets have been entered in the data base. Additionally, the annotation of each RID-set has been cross-checked and validated with the corresponding histological findings.

\section{Discussion}

The use of a complete and unified digital annotation mechanism is capable to support and enhance the process of mammography examinations and screening by unifying the interfaces between the different steps, such as image acquisition, image analysis, description of findings and documentation. Furthermore, using the described annotation tool, a complete, formal and machine-readable reference database for mammography can be obtained.

\section{References}

1. Krug B, et al. Vergleich der digitalen direkten Flachdetektor- und der analogen FilmFolien-Technik in der Darstellung normaler anatomischer Strukturen der weiblichen Brust. Geburtsh Frauenheilk 2006;66:171-178.

2. Dashmahapatra S, et al. Facilitating multi-disciplinary knowledge-based support for breast cancer screening. Int J Healthc Tech Management 2006;7(5):403-20.

3. Niemann H, Sagerer G, Schröder S, Kummert F. ERNEST: A semantic network system for pattern understanding. IEEE PAMI 1990;12(9):883 - 905.

4. American College of Radiology. Breast Imaging Reporting and Data System (BIRADS $^{\circledR}$ ) Atlas; 2006. 